\title{
Regulation of cell survival by HUNK mediates breast cancer resistance to HER2 inhibitors
}

\author{
Elizabeth S. Yeh • Melissa A. Abt • Elizabeth G. Hill
}

Received: 17 June 2014/ Accepted: 1 December 2014/Published online: 17 December 2014

(C) The Author(s) 2014. This article is published with open access at Springerlink.com

\begin{abstract}
Breast cancer patients who are HER2-positive receive targeted inhibitors to HER2, including trastuzumab and lapatinib. While patients benefit from the use of HER2 inhibitors, many fail therapy and almost all patients become resistant to treatment, indicating a critical need to prevent treatment failure. Several recent studies indicate that activation of autophagy contributes to trastuzumab and lapatinib resistance and demonstrate that impairing autophagy in breast cancer cells is therapeutically beneficial. Moreover, autophagy is mechanistically linked through signaling crosstalk to apoptotic pathways, where activation of one process impacts the other. Therefore, understanding the molecular mechanisms that control these processes may uncover novel areas of therapeutic intervention to combat or prevent resistance in breast cancer. We previously characterized the protein kinase HUNK as a breast cancer-promoting factor in HER2/neu-induced mammary tumor models, in which HUNK supported the survival of HER2/neu-positive tumor cells, likely through the regulation of apoptosis. Because significant crosstalk exists between apoptotic and autophagy proteins, we now examine if HUNK is also able to regulate cell survival
\end{abstract}

Electronic supplementary material The online version of this article (doi:10.1007/s10549-014-3227-9) contains supplementary material, which is available to authorized users.

\section{E. S. Yeh $(\bowtie) \cdot$ M. A. Abt}

Department of Cell and Molecular Pharmacology and

Experimental Therapeutics, Medical University of South

Carolina, 173 Ashley Ave, BSB358, MSC509, Charleston,

SC 29425, USA

e-mail: yeh@musc.edu

E. G. Hill

Department of Public Health Sciences, Medical University of South Carolina, Charleston, SC, USA through modulation of autophagy using HER2 inhibitor sensitive and resistant breast cancer models. Furthermore, we investigate whether inhibiting HUNK impairs in vivo tumor growth that is initiated by HER2 inhibitor-resistant breast cancer cells. Our findings indicate that therapeutically targeting HUNK is a potential strategy for overcoming resistance and that resistant breast cancer cells maintain HUNK expression to drive tumorigenesis, an observation that is consistent with a pro-survival role for this kinase.

Keywords HER2+ · Trastuzumab - Lapatinib ·

Resistance $\cdot$ HUNK $\cdot$ Breast

\section{Introduction}

Hormonally Upregulated Neu-associated kinase is a member of the AMPK-family of protein kinases that was discovered in a RT-PCR-based screen to identify kinases expressed in the adult mammary gland [1]. Previous findings indicate that HUNK is preferentially expressed in aggressive subsets of breast cancer and may play a pivotal role in mediating breast cancer metastasis [2, 3]. Consistent with these assertions, our previous work shows that HUNK expression is regulated in response to HER2/neu activity and inhibiting this kinase impairs tumor formation and growth in primary HER2/neu tumor models [4].

Although little is known about the intracellular function of HUNK, evidence is mounting that this kinase participates in the regulation of cell survival. Studies demonstrate that impairing HUNK in HER2/neu-positive (HER2+) and Akt-dependent mammary tumor models induces cell death by apoptosis $[4,5]$. Moreover, studies using Hunk ${ }^{-1-}$ (Hunk KO) mice show that normal mammary gland 
development is altered by loss of HUNK function during postlactational involution, a stage of mammary gland development governed by apoptotic clearance of mammary epithelial cells, where Hunk $K O$ mice display increased levels of apoptosis during involution [5].

The process of autophagy has been linked to apoptosis [6], and we have previously shown that HUNK mediates apoptosis $[4,5]$. However, a role for HUNK in autophagy has not been investigated. Because significant crosstalk exists between signaling pathways that regulate apoptosis and autophagy, in this study, we aimed to demonstrate that HUNK regulates autophagy in a manner consistent with its ability to regulate cell survival and show that the outcome of this activity impacts breast cancer resistance to HER2targeted therapy.

\section{Materials and methods}

Cell culture

All cells were maintained at $37{ }^{\circ} \mathrm{C}$ and $5 \% \mathrm{CO}_{2}$. Hunk $k^{+/+}$ (Hunk WT)and Hunk ${ }^{-1}$ (Hunk KO) mammary gland fibroblasts (MGF) were isolated as previously described [5] and were grown in DMEM (Hyclone) supplemented with $10 \%$ super calf serum (SCS, Gemini). BT474 (ATCC) human breast cancer cells were grown in RPMI-1640 (Hyclone) supplemented with $10 \%$ fetal bovine serum (FBS, Gibco). BT474 cells expressing control or HUNK shRNA (gift from Lewis Chodosh, University of Pennsylvania) were generated and maintained as previously described [4]. JIMT-1 (Addex Bio) trastuzumab-resistant breast cancer cells were grown in DMEM (Hyclone) supplemented with $10 \%$ FBS. JIMT-1 cells expressing control or HUNK shRNA were generated using the pGIPZ system (Thermo-GE/Dharmacon) and maintained in media containing $1 \mathrm{ug} / \mathrm{ml}$ puromycin. All media contained $2 \mathrm{mM}$ glutamine (Thermo Scientific) and Penicillin/Streptomycin (Pen/Strep, Thermo Scientific) unless otherwise specified. pEGFP-LC3 was acquired through Addgene (plasmid \#24920, provided by TorenFinkel [7] ). Transfection of GFP-LC3 was performed using Turbofect (Thermo Scientific).

\section{Immunoblotting}

Cells were lysed in buffer containing final concentrations of $50 \mathrm{mM}$ Tris- $\mathrm{HCl}, \mathrm{pH} 7.5 ; 150 \mathrm{mM} \mathrm{NaCl} ; 1 \%$ Triton $\mathrm{X}-100$; and $0.1 \%$ SDS supplemented with HALT protease and phosphatase inhibitor cocktail (Thermo Scientific). For near-infrared imaging (Odyssey, LI-COR), secondary antibodies were purchased from Rockland Scientific. Primary antibodies used for western blotting are anti-LC3B
(Cell Signaling- 2775), anti-HUNK [4], and anti- $\beta$-tubulin (Santa Cruz).

RNA isolation and quantitative Real-Time PCR

RNA was prepared using the GeneJet RNA isolation kit (Thermo Scientific). Reverse transcription was performed using the Maxima First Strand cDNA Synthesis Kit for RTPCR (Thermo Scientific). The resulting cDNA was used to perform quantitative Real-Time PCR (QRT-PCR) using the Biorad myIQ system. Primers are HUNK-For-ATTAGCT TCCTGGAGGGGAC; HUNK-Rev-GTGATATTGGGGT GCGGAT and GAPDH-For-TGCACCACCAACTGCTT AGC; GAPDH-Rev-GGCATGGACTGTGGTCATGAG

Cell death analysis

Equal numbers of cells were plated and treated the following day by serum deprivation for $24 \mathrm{~h}$ or with $100 \mathrm{nM}$ lapatinib (Santa Cruz) for $24 \mathrm{~h}$ prior to analysis by Caspase-3 activity assay (Sigma) or trypan blue exclusion and cell counting, which was performed using the cellometer mini cell counter (Nexcelom). For autophagy, cells were treated with $100 \mathrm{uM}$ chloroquine (Sigma) for the indicated times and analyzed by Western blotting or microscopy (Nikon E800).

In vivo tumorigenesis

Animal care and all animal experiments were performed with the approval and in accordance with the guidelines of the Medical University of South Carolina IACUC. For orthotopic tumor analysis, $5 \times 10^{6}$ cells were injected in the abdominal mammary fat pat of immunocompromised mice $\left(\mathrm{Nu} / \mathrm{J}-\mathrm{Foxn} 1^{\mathrm{nu} / \mathrm{nu}}\right.$, Jackson Labs). Tumors were evaluated by manual palpation using calipers.

Statistical considerations for animal data

Tumor volume data from mouse experiments were analyzed using generalized estimating equations (GEEs) $[8,9]$ with an identity link function and exchangeable correlation structure, a regression model that accommodates the lack of independence among measures obtained from the same mouse over time. Specifically, we modeled volume $\left(\mathrm{mm}^{3}\right)$ as a function of animal group (control versus HUNK shRNA), days from cell injection (measured as a continuous variable), and their two-way interaction. We used restricted cubic splines [10] to transform days from cell injection due to observed non-linear trends in tumor volume over time. Comparisons between groups at each time point were performed using model-based linear contrasts. We used Kaplan-Meier survival curves to estimate group- 
specific median time to sacrifice, and compared survival times using a log-rank test. We used a similar approach to compare time to tumor volume of $100 \mathrm{~mm}^{3}$. Analyses of animal data were performed using the $\mathrm{R}$ statistical software package [11]. GEEs were fit using the geepack library in $\mathrm{R}$ [12]. Kaplan-Meier curves and log-rank tests were conducted using the survival library in $\mathrm{R}$ [13].

\section{Results}

Autophagy is impaired in Hunk-deficient cells

We previously demonstrated that HUNK regulates cell survival $[4,5]$. Our findings indicated that impairing HUNK in mammary epithelial, mammary tumor, and breast cancer cells results in an increase in the number of cleaved-caspase-3 positive cells in mammary gland and tumor models [4, 5], suggesting that inhibiting this kinase results in cell death via apoptosis. To further investigate the role of HUNK in cell survival, we examined MGF isolated from Hunk WT and Hunk KO mice for survival response. Equal numbers of Hunk WT and Hunk KO MGF were plated and then assessed by trypan blue exclusion. Consistent with our previous findings that HUNK-deficient cells are survival impaired, Hunk KO MGF exhibited decreased numbers of viable cells after plating (Fig. 1a).

Because the signaling pathways that govern apoptosis affect a cell's ability to undergo autophagy [6], we wanted to ask if loss of HUNK alters autophagy. To evaluate autophagy response, we performed Western bot analysis for LC3B processing from LCBI to LCBII to detect levels of LC3BII formation. Equal numbers of Hunk WT and Hunk $K O$ cells were plated and the following day treated with vehicle (water) or the autophagy inhibitor, chloroquine for $4 \mathrm{~h}$. We used chloroquine because it is an autophagy inhibitor that impairs autophagic flux. Consequently, chloroquine treatment results in an accumulation of autophagosomes which incorporate LC3BII, if autophagy is proceeding as expected. Following chloroquine treatment of the Hunk WT and Hunk KO cells, we assessed for LC3BII levels by immunoblotting using the Odyssey imaging system. We found that chloroquine-treated Hunk $K O$ cells did not accumulate LC3BII when compared to Hunk WT cells, which had increased LC3BII levels in response to chloroquine treatment (Fig. 1b), suggesting that in Hunk $K O$ cells basal autophagy is likely impaired.

We confirmed these results by monitoring autophagy by GFP-LC3 to quantitate accumulation of GFP-puncta. Equal numbers of Hunk WT and Hunk KO cells were transfected with GFP-LC3 and treated with chloroquine the following day for $4 \mathrm{~h}$. Cells were then evaluated by immunofluorescence (IF) for the average number of GFP-positive puncta
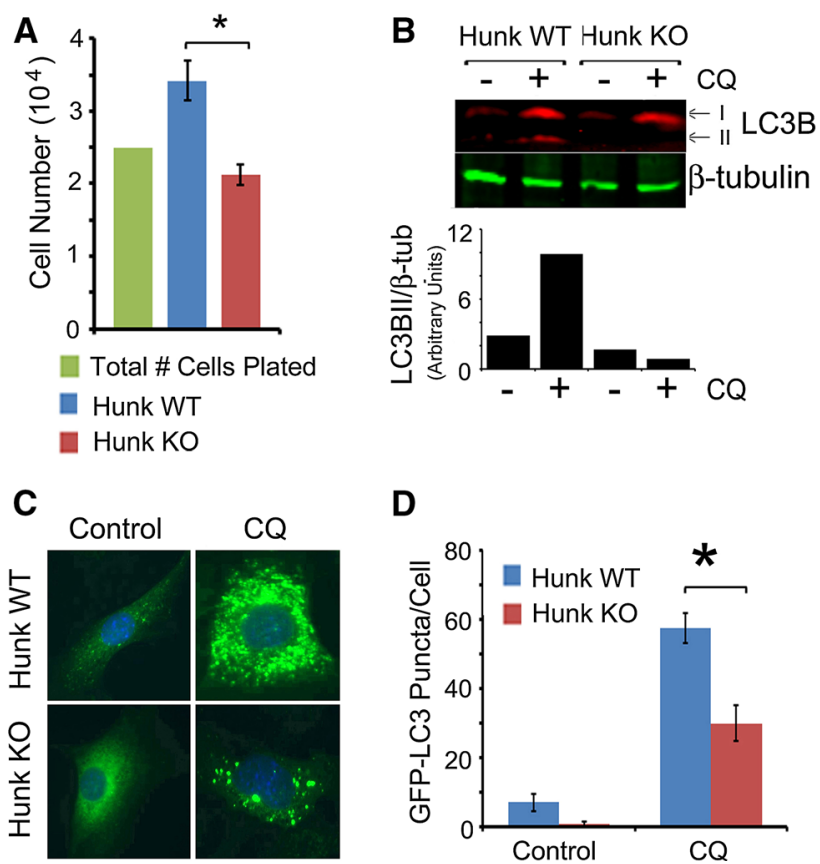

Fig. 1 HUNK promotes cell survival and regulates autophagy a Equal numbers of Hunk WT and Hunk KO MGF were plated in quadruplicate into normal media and counted $24 \mathrm{~h}$ later. ${ }^{*} p<0.001$ (Student's $t$ test). b Equal numbers of Hunk WT and Hunk KO MGF were plated and the following day treated with vehicle (water) or $100 \mathrm{uM}$ chloroquine for $4 \mathrm{~h}$. Resulting lysates were then immunoblotted for LC3BI and LC3BII levels using anti-LC3 Bantibody c Equal numbers of Hunk WT and Hunk KO MGF were seeded onto glass coverslips in triplicate and transfected with pcDNA-GFP-LC3. The following day, cells were treated with vehicle (water) or $100 \mathrm{uM}$ chloroquine for $4 \mathrm{~h}$, fixed, and stained with DAPI. d GFP-LC3 positive puncta were quantitated and averaged in at least 3 fields per slide, for 3 separate experiments. ${ }^{*} p<0.01$ (Student's $t$ test)

per cell. We found that Hunk $K O$ cells exhibited a reduced number of GFP-LC3 puncta per cell under chloroquine treatment conditions (Fig. 1c-IF, Fig. 1d-quantitation) indicating that loss or impairment of HUNK alters autophagy response. Moreover, these results are consistent with our immunoblotting results and suggest that in the absence of HUNK, autophagy is impaired.

HUNK down-regulation in primary HER2-positive breast cancer cells induces cell death in response to lapatinib and alters autophagy response

Because we previously demonstrated that HUNK is upregulated by HER2/neu and acts as a tumor-promoting factor in HER2 + breast cancer [4], we next sought to examine cell death and autophagy response in HER2+ breast cancer cells. Therefore, we used BT474 cells engineered to express control shRNA or shRNA targeted to HUNK (Fig. 2a) and examined the cells for survival response. Consistent with what we previously reported [4], HUNK shRNA-expressing BT474 cells had increased 
A
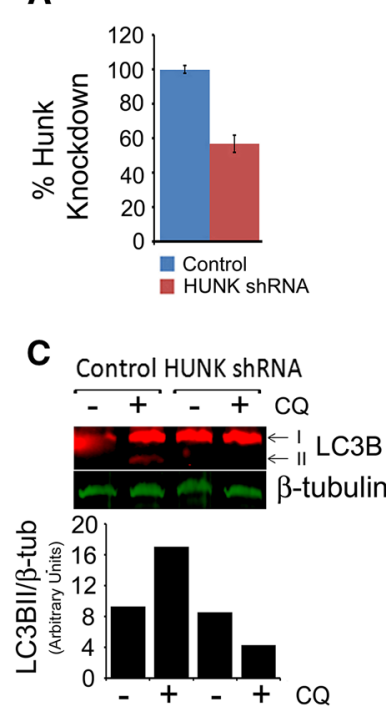

B

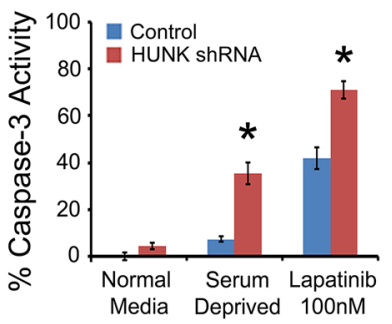

D Control HUNK ShRNA
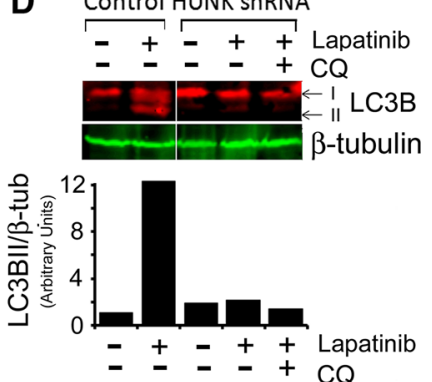

Fig. 2 HUNK knockdown in BT474 cells sensitizes cells to apoptosis and impairs autophagy a Quantitative Real-Time PCR (QRTPCR) analysis of HUNK expression levels in BT474 cells engineered to express non-functional control shRNA or shRNA targeted to HUNK. b Equal numbers of control and HUNK shRNA-expressing BT474 were plated and either maintained in normal serum, washed in PBS, and fed with no serum containing media, or treated with $100 \mathrm{nM}$ lapatinib. $24 \mathrm{~h}$ later, cells were analyzed for caspase-3 activity. Experiments performed in quadruplicate. ${ }^{*} p<0.01$ (Student's $t$ test). (c) Equal numbers of control and HUNK shRNA-expressing BT474 were plated. The following day, cells were treated with vehicle (water) or $100 \mathrm{uM}$ chloroquine for $4 \mathrm{~h}$ and analyzed by immunoblotting for LC3BI and LC3BII. d Equal numbers of control and HUNK shRNA-expressing BT474 were plated. The following day cells were treated with vehicle (DMSO) or $100 \mathrm{nM}$ lapatinib for $24 \mathrm{~h}$. Cells treated with lapatinib plus chloroquine received $100 \mathrm{uM}$ chloroquine for $4 \mathrm{~h}$ prior to lysis. Lysates were analyzed by immunoblotting for LC3BI and LC3BII. Quantitation is LC3BII/ $\beta$ tubulin

levels of caspase-3 activity in response to serum withdrawal (Fig. 2b), confirming that HUNK promotes cell survival. To determine if HUNK mediates cell death in response to HER2 inhibition, we treated control and HUNK shRNA-expressing BT474 cells with lapatinib and measured caspase- 3 activity. We found that indeed, HUNK shRNA-expressing BT474 cells were more sensitive to lapatinib, resulting in higher levels of caspase-3 activity (Fig. 2b).

We next evaluated levels of basal autophagy in control and HUNK shRNA-expressing BT474 cells. Equal numbers of each cell type were plated and the following day treated with chloroquine for $4 \mathrm{~h}$. Following, cells were assessed for levels of LC3BI processing to LC3BII. Similar to our findings in Fig. 1b, we saw an increase in LC3BII in control cells treated with chloroquine but this did not occur in HUNK shRNA-expressing BT474 cells (Fig. 2c).

Previously, it was determined that HER2+ breast cancer cells respond to HER2 inhibition by inducing autophagy as a mechanism to survive inhibitor treatment [14-17]. Therefore, we next examined levels of LC3BI processing to LC3BII in control and HUNK shRNA-expressing BT474 cells that had been treated with lapatinib. Similar to previously published results [16], we saw an increase in LC3BII levels in control cells treated with lapatinib (Fig. 2d, compare lane 1 to lane 2) but this increase in LC3BII levels was not induced in HUNK shRNA BT474 cells treated with lapatinib (Fig. 2d, compare lane 3 to lane 4). To further evaluate the observed lack of LC3BII processing, and thus possible diminished autophagy response to lapatinib in HUNK shRNA cells, we additionally cotreated HUNK shRNA cells with chloroquine and lapatinib to assess LC3BII levels. Consistent with our observations thus far, chloroquine treatment did not result in an accumulation of LC3BII in HUNK shRNA cells also treated with lapatinib (Fig. 2d, compare lane 4 to lane 5). Moreover, there was no difference in LC3BII levels between HUNK shRNA cells treated with vehicle, lapatinib alone, and those cell that had been treated with both lapatinib and chloroquine (Fig. 2d, compare lanes 3, 4, and 5), implying that autophagy is impaired due to HUNK deficiency in BT474 HER2+ breast cancer cells.

HUNK regulates cell survival in breast cancer cells resistant to HER2-targeted therapy

As autophagy is induced by HER2+ breast cancer cells in response to HER2 inhibition as a means to promote survival, it has been suggested that autophagy is a mechanism for developing resistance to HER2 inhibitors [14-17]. Therefore, we next wanted to ask if targeting HUNK in cells that are resistant to HER2 inhibitor also alters cell survival response. Therefore, we examined the JIMT-1 breast cancer cell line [18] for autophagy response by LC3B processing. JIMT-1 cells were isolated from a trastuzumab resistant patient and are insensitive to HER2 and EGFR inhibitors including lapatinib [18, 19].

We engineered JIMT-1 cells to express control or HUNK shRNA (Fig. 3a).To evaluate autophagy, equal numbers of control and HUNK shRNA-expressing JIMT-1 cells were plated and treated the following day with chloroquine for $4 \mathrm{~h}$. Following, samples were evaluated for LC3BII levels by Western blotting. In agreement with our findings in Fig. 1, HUNK shRNA-expressing JIMT-1 cells also had impaired LC3BII processing in the presence of chloroquine, whereas control cells accumulated LC3BII under the same treatment conditions (Fig. 3b). We then evaluated these cells for viability. Similar to our findings in Fig. 1, we found that HUNK shRNA-expressing JIMT-1 cells had reduced viability (Fig. 3c) and increased cell 
A

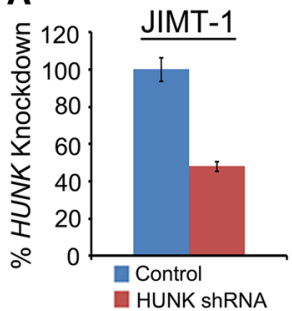

C

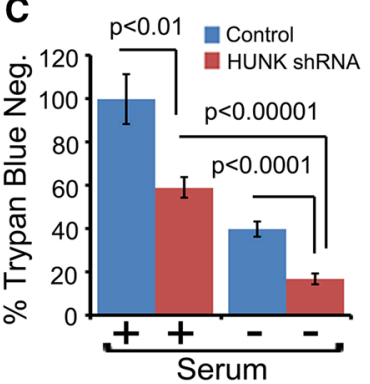

B

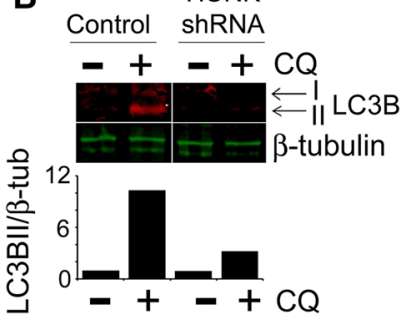

D

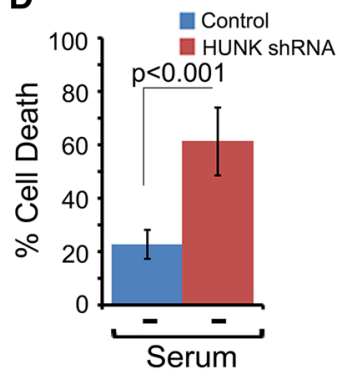

Fig. 3 HUNK knockdown in trastuzumab-resistant JIMT-1 cells impairs autophagy and impacts survival. a Quantitative Real-Time PCR (qRT-PCR) analysis of HUNK expression levels in JIMT-1 cells engineered to express non-functional control shRNA or shRNA targeted to HUNK. b Equal numbers of control and HUNK shRNAexpressing JIMT-1 cells were plated. The following day, cells were treated with vehicle (water) or $100 \mathrm{uM}$ chloroquine for $4 \mathrm{~h}$ and analyzed by immunoblotting for LC3BI and LC3BII. Quantitation is LC3BII/ $\beta$-tubulin. c Equal numbers of JIMT-1 control and HUNK shRNA-expressing cells were plated in quadruplicate into normal media. $18 \mathrm{~h}$ after plating, cells were washed in PBS and fed with normal- or no-serum-containing media. Cells were subsequently counted $24 \mathrm{~h}$ later and represented as the percent of cells that were trypan blue negative.p values as indicated. (Student's $t$ test). d Quantitation of percent of dead cells from (c). $p<0.001$ (Student's $t$ test)

death (Fig. 3d) in response to serum withdrawal when compared to control JIMT-1 cells. Taken together, our data suggest that targeting HUNK in HER2+ primary and resistant breast cancer cells induces cell death and impacts the ability of these cells to undergo autophagy.

Targeting HUNK impairs tumor initiation and growth in vivo of breast cancer cells resistant to HER2-targeted therapy

To determine if the effect of targeting HUNK on cell death and autophagy has an effect on tumorigenesis of breast cancer cells resistant to HER2-targeted therapy, we next assessed tumorigenesis in vivo. Equal numbers of control or HUNK shRNA-expressing JIMT-1 cells were introduced into the abdominal mammary gland of immunocompromised mice and monitored for tumor formation and growth over time. As predicted by the in vitro findings we report here, targeting HUNK in JIMT-1 cells impaired tumor growth (Fig. 4a), where the average tumor volume in the HUNK shRNA group was significantly reduced from day 24 after injection onward (summarized in Table 1).

A

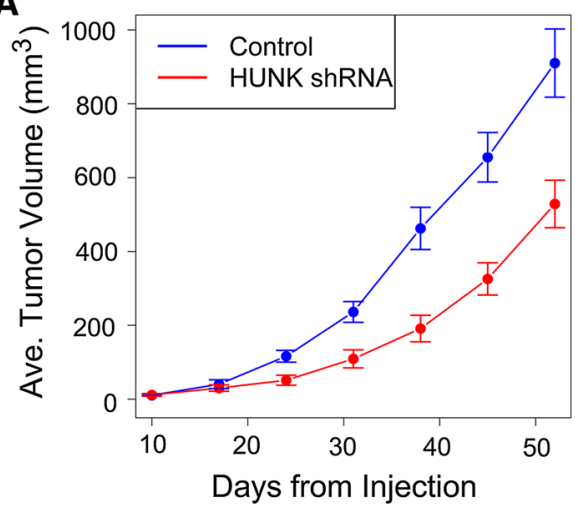

B

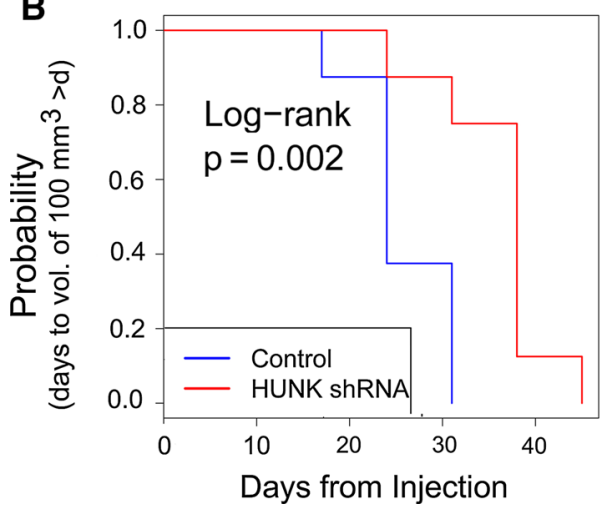

Fig. 4 HUNK knockdown in trastuzumab-resistant JIMT-1 cells impairs mammary tumorigenesis in vivo. a $5 \times 10^{6}$ control or HUNK shRNA-expressing JIMT-1 cells were injected into the abdominal mammary fat pad of immunocompromised nude mice and monitored for tumor volume over time. Average tumor volume and corresponding standard errors at each time point, $n=8$ mice per group. Statistical analysis was performed as indicated in the Methods section. b Time to tumor size of $100 \mathrm{~mm}^{3}$ was significantly longer for HUNK shRNA mice relative to control mice based on Kaplan-Meier survival curve estimates; $p=0.002, \log$-rank test. Statistical analysis was performed as indicated in the Methods section

Table 1 Analysis of average tumor volume at indicated times postinjection

\begin{tabular}{llcc}
\hline Day & $\begin{array}{l}\text { Control average } \\
\text { volume (SE) }\end{array}$ & $\begin{array}{l}\text { HUNK shRNA average } \\
\text { volume (SE) }\end{array}$ & $p$ \\
\hline 10 & $10.6(2.3)$ & $11.6(3.6)$ & 0.71 \\
17 & $40.5(12.0)$ & $30.1(8.6)$ & 0.93 \\
24 & $116.3(16.0)$ & $51.3(13.5)$ & $<0.0001$ \\
31 & $236.4(57.2)$ & $109.3(24.4)$ & $<0.0001$ \\
38 & $462.4(57.2)$ & $191.3(35.9)$ & $<0.0001$ \\
45 & $655.4(67.0)$ & $325.7(43.6)$ & $<0.0001$ \\
52 & $910.3(92.4)$ & $528.7(64.4)$ & $<0.0001$ \\
\hline
\end{tabular}

Analysis includes standard error values and $p$ value at each time point, $n=8$ mice per group at each time point indicated. Statistical analysis was performed as indicated in the methods section

Furthermore, impairing HUNK in the resistant cells improved overall survival time. Kaplan-Meier survival curve estimate of median time to sacrifice was significantly 
increased in the HUNK shRNA cohort (67 days HUNK shRNA vs. 57.5 days control shRNA, $p=0.005$ ). Additionally, the median time for HUNK-deficient tumors to reach a volume of $\sim 100 \mathrm{~mm}^{3}$ was significantly delayed (Fig. 4b, 38 days HUNK shRNA vs. 24 days control shRNA, $p=0.002$ ).

Despite an obvious delay in tumor initiation, HUNK shRNA-derived tumors began to grow at a similar rate as the control tumors, an observation supported by parallel tumor growth curves when displayed using a semi-log plot (Supplementary Fig. 1a). Therefore, we analyzed the resulting tumors that were collected upon completion of our in vivo analysis to re-examine HUNK expression levels. We found that HUNK levels were similar by QRTPCR (Supplementary Fig. 1b) and by immunoblotting (Supplementary Fig. 1c) in the control and HUNK shRNA tumor groups, despite the fact that JIMT-1 cells containing HUNK shRNA were initially knocked down for HUNK (Fig. 3a). This finding suggests that there is likely a selective pressure for the HER2 inhibitor-resistant JIMT-1 tumor cells to reject or overcome HUNK knockdown by shRNA and maintain HUNK expression for survival.

Taken together, our in vivo finding that inhibiting HUNK in resistant JIMT-1 breast cancer cells impairs tumor growth is in line with our in vitro findings that HUNK regulates cell survival and modulates autophagy. Furthermore, our observation that a selective pressure for tumor cells to maintain HUNK expression as a means to promote tumor growth is consistent with our findings that HUNK promotes tumor cell survival by regulating apoptosis and autophagy. As a whole, the observations we present suggest that targeting HUNK in breast cancers resistant to HER2-targeted inhibitors has the potential to be therapeutically beneficial.

\section{Discussion}

Although a role for HUNK in promoting breast cancer initiation $[4,5]$ and metastasis $[2,3]$ has been uncovered, little is known about the intracellular function of this kinase. Evidence is mounting for a role for HUNK in regulating cell survival. Previous findings indicate that HUNK regulates apoptosis [4, 5]. We now report that HUNK also regulates cell survival via autophagy. This finding is significant because autophagy is a mechanism by which breast cancer cells have been shown to survive treatment and acquire resistance $[14,17,20]$. However, to some degree, the role of autophagy in human cancer is still unclear as this process is reported as both tumor promoting [14, 17, 20-25] and tumor inhibitory [26-28]. This paradox is exemplified in HER2+ breast cancer in which monoallelic deletion of the autophagy gene $B E C N-1$ is frequently found yet these tumors are more sensitive to HER2-targeted therapy [29]. This suggests that during early stages of tumor development, autophagy is tumor inhibitory but once a tumor is fully developed inhibiting autophagy makes treatment much more effective. Consequently, to clarify how to therapeutically target autophagy in breast cancer, carefully mapping the cell signaling pathways and molecules that control these processes is critical to develop effective breast cancer therapies.

Consistent with the idea that autophagy status influences tumor cell survival in response to treatment, autophagy competent tumor cells will adapt by utilizing autophagy to survive treatment, thus acquiring resistance. Therefore, being able to simultaneously induce tumor cell death by apoptosis and impair survival by autophagy has the potential to allow tumor cells to evade resistance. This strategy is directly applicable to HER2+ breast cancer because chronic application of HER2 inhibitors (i.e., trastuzumab and lapatinib) has been shown to lead to the up-regulation of autophagy response [14, 17] and survival signaling pathways, like the PI3K/Akt pathway, in breast cancer cells to promote cell survival [30]. Our new findings implicate HUNK as an attractive target because this kinase not only signals through HER2 [4] and PI3K/Akt [5] but we now show HUNK also regulates cell survival by impacting both apoptosis and autophagy.

While intriguing, additional investigation is warranted as we do not yet know the mechanism by which HUNK regulates autophagy and how this relates to apoptosis. It is possible that HUNK regulates these processes individually or alternatively and mediates crosstalk between the two pathways. We have previously demonstrated that HUNK can regulate p27 downstream of HER2 activation and attribute HUNK's ability to promote survival, at least in part due to this function [4]. Interestingly, p27 has been implicated in autophagy, and this function of $\mathrm{p} 27$ is directed by its phosphorylation at Threonine (T) 198 [31]. The phosphorylation of p27 on T198 can be directed by multiple kinases including AMPK [31], Akt [32], and Rsk1 [33]. AMPK-directed phosphorylation of p27 is associated with p27's function toward autophagy [31] whereas phosphorylation of p27 by Akt mediates cell survival [32]. We have shown that HUNK is upregulated by Akt [5]. Accordingly, it is possible that HUNK could have a selective function toward Akt-specific downstream signaling molecules, and it will be interesting to determine if HUNK regulates p27 in an Akt-dependent manner. Furthermore, it is perhaps appealing to speculate that an overlapping mechanism for HUNK exists toward Akt-specific substrates such as transcriptional regulation, modulation of protein localization, or sequestration of proteins in inactive complexes-perhaps by HUNK-directed phosphorylation. However, very little is known about the kinase activity of HUNK or its preferred 
phosphorylation consensus site. Consequently, this line of investigation is better suited for future studies.

In conclusion, our collective findings to date demonstrate that HUNK is a critical survival protein in HER2+ breast cancer cells. We have shown that HUNK regulates tumor cell survival by mediating apoptosis, and now we have also demonstrated that HUNK regulates survival by mediating autophagy. Taken together, these findings implicate HUNK as a potential target in HER2+ breast cancer that has the potential to be therapeutically beneficial in fighting resistance to HER2-targeted therapies.

Acknowledgments The Yeh lab is supported by start-up funding from Medical University of South Carolina, by pilot research funding from an American Cancer Society Institutional Research Grant (IRG97-219-14) from the American Cancer Society awarded to the Hollings Cancer Center, Medical University of South Carolina, by pilot research funding from a Department of Defense grant (W81XWH-112-0229) at the Medical University of South Carolina, and by an award from the Concern Foundation. These studies were also supported by the Biostatistics Shared Resource, Hollings Cancer Center, Medical University of South Carolina (P30 CA138313). ESY designed experiments, performed experiments, and wrote the paper. MAA performed experiments. EGH performed the statistical analysis of animal data and wrote the corresponding statistical methods section.

Conflict of interest The authors declare that they have no conflicts of interest.

Open Access This article is distributed under the terms of the Creative Commons Attribution Noncommercial License which permits any noncommercial use, distribution, and reproduction in any medium, provided the original author(s) and the source are credited.

\section{References}

1. Chodosh LA, Gardner HP, Rajan JV, Stairs DB, Marquis ST, Leder PA (2000) Protein kinase expression during murine mammary development. Dev Biol 219(2):259-276

2. Wertheim GB, Yang TW, Pan TC, Ramne A, Liu Z, Gardner HP, Dugan KD, Kristel P, Kreike B, van de Vijver MJ et al (2009) The Snf1-related kinase, Hunk, is essential for mammary tumor metastasis. Proc Natl Acad Sci USA 106(37):15855-15860

3. Quintela-Fandino M, Arpaia E, Brenner D, Goh T, Yeung FA, Blaser H, Alexandrova R, Lind EF, Tusche MW, Wakeham A et al (2010) HUNK suppresses metastasis of basal type breast cancers by disrupting the interaction between PP2A and cofilin-1. Proc Natl Acad Sci USA 107(6):2622-2627

4. Yeh ES, Yang TW, Jung JJ, Gardner HP, Cardiff RD, Chodosh LA (2011) Hunk is required for HER2/neu-induced mammary tumorigenesis. J Clin Invest 121(3):866-879

5. Yeh ES, Belka GK, Vernon AE, Chen CC, Jung JJ, Chodosh LA (2013) Hunk negatively regulates c-myc to promote Akt-mediated cell survival and mammary tumorigenesis induced by loss of Pten. Proc Natl Acad Sci USA 110(15):6103-6108

6. Maiuri MC, Zalckvar E, Kimchi A, Kroemer G (2007) Self-eating and self-killing: crosstalk between autophagy and apoptosis. Nat Rev Mol Cell Biol 8(9):741-752

7. Lee IH, Cao L, Mostoslavsky R, Lombard DB, Liu J, Bruns NE, Tsokos M, Alt FW, Finkel T (2008) A role for the NAD- dependent deacetylase Sirt1 in the regulation of autophagy. Proc Natl Acad Sci USA 105(9):3374-3379

8. Liang K, Zeger S (1986) Longitudinal data analysis using generalized linear models. Biometrika 73:13-22

9. Zeger SL, Liang KY (1986) Longitudinal data analysis for discrete and continuous outcomes. Biometrics 42(1):121-130

10. Stone CA, Koo CY (1985) Additive splines in statistics. Proc Stat Comput Sect ASA 27:45-58

11. RCoreTeam: R (2012) A language and environment for statistical computing. In: R Foundation for Statistical Computing, Vienna. ISBN 3-900051-07-0. http://www.R-project.org. Accessed 25 Nov 2014

12. HØjsgaard S, Halekoh U, Yan J (2006) The R package geepack for generalized estimating equations. J Stat Softw 15:1-11

13. Therneau $\mathrm{T}$ (2014) A package for survival analysis in S. R package version 2.37-7. http://www.CRANR-projectorg/package=survival 2014. Accessed 25 Nov 2014

14. Chen S, Li X, Feng J, Chang Y, Wang Z, Wen A (2011) Autophagy facilitates the Lapatinib resistance of HER2 positive breast cancer cells. Med Hypotheses 77(2):206-208

15. Cufi S, Vazquez-Martin A, Oliveras-Ferraros C, Corominas-Faja B, Urruticoechea A, Martin-Castillo B, Menendez JA (2012) Autophagy-related gene 12 (ATG12) is a novel determinant of primary resistance to HER2-targeted therapies: utility of transcriptome analysis of the autophagy interactome to guide breast cancer treatment. Oncotarget 3(12):1600-1614

16. Cufi S, Vazquez-Martin A, Oliveras-Ferraros C, Corominas-Faja B, Cuyas E, Lopez-Bonet E, Martin-Castillo B, Joven J, Menendez JA (2013) The anti-malarial chloroquine overcomes primary resistance and restores sensitivity to trastuzumab in HER2positive breast cancer. Sci Rep 3:2469

17. Vazquez-Martin A, Oliveras-Ferraros C, Menendez JA (2009) Autophagy facilitates the development of breast cancer resistance to the anti-HER 2 monoclonal antibody trastuzumab. PLoS ONE 4(7):e6251

18. Tanner M, Kapanen AI, Junttila T, Raheem O, Grenman S, Elo J, Elenius K, Isola J (2004) Characterization of a novel cell line established from a patient with Herceptin-resistant breast cancer. Mol Cancer Ther 3(12):1585-1592

19. Koninki K, Barok M, Tanner M, Staff S, Pitkanen J, Hemmila P, Ilvesaro J, Isola J (2010) Multiple molecular mechanisms underlying trastuzumab and lapatinib resistance in JIMT-1 breast cancer cells. Cancer Lett 294(2):211-219

20. Cook KL, Shajahan AN, Clarke R (2011) Autophagy and endocrine resistance in breast cancer. Expert Rev Anticancer Ther 11(8):1283-1294

21. Chaachouay H, Ohneseit P, Toulany M, Kehlbach R, Multhoff G, Rodemann HP (2011) Autophagy contributes to resistance of tumor cells to ionizing radiation. Radiotherapy and oncology. J Eur Soc Ther Radiol Oncol 99(3):287-292

22. Moussay E, Kaoma T, Baginska J, Muller A, Van Moer K, Nicot N, Nazarov PV, Vallar L, Chouaib S, Berchem G et al (2011) The acquisition of resistance to TNFalpha in breast cancer cells is associated with constitutive activation of autophagy as revealed by a transcriptome analysis using a custom microarray. Autophagy $7(7): 760-770$

23. Schoenlein PV, Periyasamy-Thandavan S, Samaddar JS, Jackson WH, Barrett JT (2009) Autophagy facilitates the progression of ERalpha-positive breast cancer cells to antiestrogen resistance. Autophagy 5(3):400-403

24. Sun WL, Chen J, Wang YP, Zheng H (2011) Autophagy protects breast cancer cells from epirubicin-induced apoptosis and facilitates epirubicin-resistance development. Autophagy 7(9):1035-1044

25. Yoon JH, Ahn SG, Lee BH, Jung SH, Oh SH (2012) Role of autophagy in chemoresistance: regulation of the ATM-mediated DNA-damage signaling pathway through activation of DNAPKcs and PARP-1. Biochem Pharmacol 83(6):747-757 
26. Liang XH, Jackson S, Seaman M, Brown K, Kempkes B, Hibshoosh H, Levine B (1999) Induction of autophagy and inhibition of tumorigenesis by beclin 1. Nature 402(6762):672-676

27. Qu X, Yu J, Bhagat G, Furuya N, Hibshoosh H, Troxel A, Rosen J, Eskelinen EL, Mizushima N, Ohsumi Y et al (2003) Promotion of tumorigenesis by heterozygous disruption of the beclin 1 autophagy gene. J Clin Investig 112(12):1809-1820

28. Yue Z, Jin S, Yang C, Levine AJ, Heintz N (2003) Beclin 1, an autophagy gene essential for early embryonic development, is a haploinsufficient tumor suppressor. Proc Natl Acad Sci USA 100(25): 15077-15082

29. Vazquez-Martin A, Cufi S, Oliveras-Ferraros C, Martin-Castillo B, Del Barco S, Lopez-Bonet E, Menendez JA (2011) Expression status of the autophagy-regulatory gene ATG6/BECN1 in ERBB2-positive breast carcinomas: bypassing ERBB2-induced oncogenic senescence to regulate the efficacy of ERBB2-targeted therapies. Genes Chromosom Cancer 50(4):284-290
30. Rexer BN, Arteaga CL (2012) Intrinsic and acquired resistance to HER2-targeted therapies in HER2 gene-amplified breast cancer: mechanisms and clinical implications. Crit Rev Oncog 17(1):1-16

31. Liang J, Shao SH, Xu ZX, Hennessy B, Ding Z, Larrea M, Kondo S, Dumont DJ, Gutterman JU, Walker CL et al (2007) The energy sensing LKB1-AMPK pathway regulates p27(kip1) phosphorylation mediating the decision to enter autophagy or apoptosis. Nat Cell Biol 9(2):218-224

32. Fujita N, Sato S, Katayama K, Tsuruo T (2002) Akt-dependent phosphorylation of p27Kip1 promotes binding to 14-3-3 and cytoplasmic localization. J Biol Chem 277(32):28706-28713

33. Fujita N, Sato S, Tsuruo T (2003) Phosphorylation of p27Kip1 at threonine 198 by p90 ribosomal protein S6 kinases promotes its binding to 14-3-3 and cytoplasmic localization. J Biol Chem 278(49):49254-49260 